\title{
Organización policial y debilidad institucional: balance de las capacidades de las policías estatales
}

\author{
Police Organization and Institutional Weakness. \\ An Assessment of State Police Capacities. \\ Arturo Alvarado Mendoza* \\ El Colegio de México \\ https://orcid.org/o000-0002-6922-3507 \\ Sergio Padilla Oñate** \\ El Colegio de México \\ https://orcid.org/oooo-000I-9528-4613
}

ISSN-OI85-4259; e- ISSN : 2007-9I76

DOI: http://dx.doi.org/I0.28928/ri/90202I/atcI/alvaradoa/padillaonates

\begin{abstract}
Resumen
Este artículo examina las reformas efectuadas para mejorar las capacidades de las policías de las entidades federativas de México durante más de una década. Aunque varios estudios han analizado la capacidad del Estado nacional en materia de seguridad pública, conocemos poco el papel que han tenido los gobiernos estatales en la construcción de dicha política. Contribuimos al tema comparando la seguridad pública de los 32 estados proponiendo un índice de capacidades por medio de la técnica de factores principales, que integra siete variables que dan cuenta de cuatro procesos de construcción institucional: la cimentación de una policía profesional; la coordinación efectiva de los gobiernos estatales con el federal, el ejercicio del gasto en seguridad y la relación con los ciudadanos. Los resultados muestran un panorama muy diverso: existen gobiernos con capacidades limitadas y vulnerables, pero hay otros que han fortalecido sus policías y mejorado la percepción de la ciudadanía.

Palabras clave: fortaleza institucional, capacidad policial, gobernanza de la seguridad, seguridad pública, México.
\end{abstract}

\footnotetext{
Abstract

The article analyses the set of reforms implemented to improve capacities in state police organizations in Mexico on more than a decade. Several studies have examined national security capacity, but little is known related to the role of state governments in the development of an effective public security system. This work contributes to the knowledge with a comparative assessment of state capacities. We build an index and apply a Principal Factor Analysis, with seven variables that account for four processes: Federal-state government relations, expenditure in security, the establishment of professional state police corps and an improvement on citizens' approval. Results shows strong disparities among states to protect their citizens. Some are still weak and vulnerable while others have made improvements, strengthen their police organizations and enhance responses to citizens.

Key words $¥$ State Police, Capacity, Police Organization, Governors, Governance in Security, México.
}

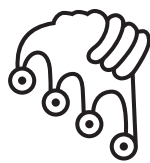

IZTAPALAPA

Agua sobre lajas

*alvarado@colmex.mx

**spadilla@colmex.mx 


\section{Introducción}

l comportamiento de las policías mexicanas en el combate a los delitos y en el
mantenimiento de un orden legal y legítimo se han convertido en una de las
mayores preocupaciones de la sociedad y de las autoridades del Estado por más de dos décadas. Las autoridades federales y estatales han realizado numerosas reformas esperando mejorar el desempeño policial. No obstante las trasformaciones, hay un claro desfase entre el crecimiento sostenido del crimen, las disímiles respuestas policiales y muchas consecuencias no previstas de los cambios. Por este motivo es pertinente preguntarse ¿cuál ha sido la eficacia de las reformas?, y ¿cuáles son los indicadores adecuados para medirla?

La literatura que estudia el tema no es concluyente sobre los logros o avances y una vertiente mayoritaria es claramente crítica de los programas al contrastarlos con el incremento de los delitos. Cierto es que continúan en aumento y que tampoco se han erradicado ni la corrupción ni los abusos policiales que motivaron los cambios, pero también muchos estudios coinciden en que la capacidad no puede medirse únicamente con base en la reducción de delitos (Stucky, 2005).

Acorde con lo anterior, nuestra propuesta consiste en una medición de las capacidades de gobierno y policía, tomando en cuenta más variables que la eficacia en el combate a los delitos, el liderazgo en seguridad o el análisis de las tensiones entre políticos y operadores, porque estas no logran reflejar el impacto de las reformas para la ciudadanía. Medir la capacidad implica evaluar el ejercicio eficaz del gasto, la profesionalización policial y la percepción ciudadana respecto del desempeño de las policías y de la política de seguridad. Esta perspectiva trasciende los estudios sobre las reformas, a la vez que permite comparar a los 32 gobiernos estatales, lo cual abre la puerta a futuras investigaciones que tomen como referencia los hallazgos que presentamos y que puedan responder preguntas más específicas sobre las fortalezas y debilidades de los gobiernos y de las policías para mejorar la seguridad de los ciudadanos. 
El artículo parte de una pregunta asociada con las capacidades de las policías de México: después de una década de reformas, ¿̧han adquirido las policías estatales ${ }^{1}$ capacidades para implantar un orden legal y legítimo, para controlar, reducir o eliminar el abuso, la corrupción y la percepción de inseguridad en sus entidades? $\mathrm{El}$ análisis abarca aspectos normativos (un marco de funciones legales coherente, basado en un estado de derecho), coercitivos (un conjunto de fuerzas policiales con entrenamiento, articulación, así como poder de disuasión, de prevención y de fuego-punición) y de control territorial (soberanía territorial en su jurisdicción).

Para responder a esta y otras interrogantes, el artículo se organiza de la siguiente manera: primero se aborda el contexto de las reformas policiales en México, poniendo énfasis en los sexenios de Felipe Calderón y Enrique Peña Nieto; en segundo lugar, se desarrolla el marco analítico y las hipótesis derivadas de este; en tercer lugar, se expone la metodología que guía la investigación; en cuarto, se discuten los resultados del índice de capacidad estatal en seguridad pública y, por último, se presentan las conclusiones.

\section{La literatura sobre las reformas policiales ${ }^{2}$}

Una buena proporción de la literatura que investiga y fomenta el debate público sobre el comportamiento policial se centra en las acciones federales (o nacionales), y abunda en críticas al diseño, la implementación y el desempeño de las fuerzas federales (el Ejército, la Marina, las diversas policías federales y la Guardia Nacional), en sus errores -algunos evidentes-, pero no en sus logros (Benítez y Aguayo, 2016; Aguayo y Dayán, 2018). Algunos trabajos ofrecen estudios sistemáticos de los programas, gracias a los cuales hemos entendido muchas de las limitaciones de la política pública nacional. Bailey (2014), por ejemplo, afirma que la respuesta del gobierno frente a los problemas ha sido inadecuada para implantar un orden legal. Amplio es el corpus dedicado al fracaso del combate a las organizaciones criminales (Correa-Cabrera, 2017; Maldonado, 2017; Durán, 2020); otros hablan de guerra y

1 Nos referimos a las policías de las 32 entidades federativas bajo el mando de los gobernadores, que incluyen preventivas y ministeriales y pueden estar unificadas, coordinadas o tener otra denominación.

2 Por reforma entendemos una política explícita iniciada por una autoridad, o un conjunto de autoridades, que propone modificar el statu quo de ciertas reglas, estructuras, organizaciones, prácticas y procedimientos burocráticos. Sus causas no están en disputa, pero deben ser examinadas en términos de su factibilidad y de resultados estratégicos. 
de formas de actuación abusivas por parte de las autoridades (Silva et al., 20I8; Silva y Padilla, 2020; Schedler, 2018). En todos los casos manifiestan cómo el crimen impactó a los gobiernos y sus policías al exhibir sus debilidades y contradicciones. Ofrecen evidencias de un descontrol de la seguridad y de un poder inexorable de las organizaciones criminales, que dominan territorios, subyugan poblaciones, doblegan o sobornan autoridades y policías en todos los niveles (Durán, 2020).

Algunos argumentan que la presencia de la delincuencia organizada no basta para explicar las fallas de las policías y se concentran en los problemas propios del campo policial para analizar las reformas, las causas que las produjeron y sus resultados, por lo demás subóptimos (Sabet, 20I2; Davis, 2006; LaRose y Maddan, 2009). Hay quienes sostienen que las políticas reactivas de débil articulación en los estados indican la ineptitud de las policías locales y su consecuente proceso de centralización federal o su militarización en detrimento de los derechos humanos y las capacidades de los estados (Padilla, 2019; Moloeznik y Suárez, 20I2; Morales y Pérez, 20I5). Otros más argumentan la debilidad y corruptibilidad “inherente" de las policías locales (Davis, 2020; Causa en Común, 2018; México Evalúa, 2018) y, por supuesto, de sus directivos nacionales.

Varios autores han abundado sobre la implementación de los programas de policía comunitaria, vecinal, de proximidad o dedicada a la solución de problemas (Ungar, 2002; Suárez, 20II). Otros han examinado el tema de las capacidades de las políticas y de los actores estatales de seguridad (Arzt, 2006; Arroyo, 2006; Alvarado, 2006; Guerrero, 20II; Suárez et al., 20IIः 5I-52; Sabet, 20I2; Luna, 20I4; Llanos et al. 2018). Algunos proponen que las reformas policiales deben estudiarse desde un modelo de análisis de las políticas públicas, mientras que otros parten de una visión sustentada en la seguridad ciudadana o en los modelos de policías comunitarias o de proximidad. En todos los casos continúan midiendo el éxito o fracaso de las reformas con parámetros parciales, o inconsistentes, que no explican el problema de operación policial dentro de organizaciones estatales complejas y multinivel. Con excepción de González (2019) y Lisón (2016), los trabajos dejan sin explicación las asimetrías y las complejas relaciones de poder entre la federación y los estados, que han sido parte de las luchas políticas y transformaciones de las instituciones de seguridad del Estado mexicano. Ahora bien, algunos han ensayado interpretaciones sobre las conductas inadecuadas o fallidas de las instituciones del Estado, pero hace falta una explicación teórica fundamentada. El enfoque comparativo de este artículo permite contextualizar mejor los estudios de caso citados.

Para justificar el estudio de las policías estatales en el proceso de reformas es pertinente considerar tres aspectos. En primer lugar, en materia de criminalidad, 
la gran mayoría de los presuntos delitos registrados son del fuero común, clasificados de acuerdo con los códigos penales de cada entidad. En segundo lugar, 82\% de las detenciones de presuntos delicuentes son efectuadas por policías estatales y municipales (las ministeriales arrestan 35\%, las estatales $15 \%$ y las municipales $32 \%$ y, en mucho menor medida participa la federación con $18 \%$ [Enpol, 2016]). Esto corresponde con la distribución de fuerzas policiales, que suman 212 I95 policías estatales y ministeriales, de un total de 362000 elementos locales, incluyendo a las municipales, que no estudiamos en este trabajo. En tercer lugar, el gasto federal en seguridad es inmenso, pero casi nunca tomamos en cuenta que el gasto policial tiene un componente estatal más importante y constituye una variable estructural de capacidad (Sánchez, 2020; México Evalúa, 20II). Los presupuestos estatales son una manifestación de los esfuerzos y preferencias de los gobernadores para combatir el crimen. Sus fuentes son aportaciones estatales y subsidios federales. ${ }^{3}$

Estos indicadores muestran que las entidades federativas desempeñan un papel central en materia de seguridad del país. No obstante, poco conocemos de sus capacidades efectivas y del impacto que han tenido más de I2 años de reformas, supuestamente para fortalecerlas. Necesitamos explicar cómo funcionan las policías estatales dentro del sistema de gobernanza del Estado en general, sus dilemas, conflictos y los retos que enfrentan en su acción. Esperamos aportar a la solución de este problema público.

\section{Las reformas y el desenvolvimiento de las policías}

Los procesos de reforma que nos interesa analizar son los implementados a partir de la presidencia de Felipe Calderón, la cual se ha estudiado desde la perspectiva de la militarización, pero poco se ha dicho sobre su proyecto de reforma policial. Durante su administración se rediseñó la Policía Federal y se creó un plan para el fortalecimiento de las policías locales (estatales y municipales) y dos fondos, el Subsidio para la Seguridad Municipal (Subsemun) y el Fondo de Aportaciones para la Seguridad Pública (FASP). La adopción de estas medidas marcó un parteaguas dentro de las políticas de seguridad a nivel nacional pues los estados y municipios comenzaron a recibir dinero de la federación para fortalecer sus policías.

3 Por ejemplo, entre 2013 y 2018 el gasto federal transferido a estados y municipios para seguridad sumó 34.5\%. del presupuesto total federal para este rubro (Causa en Común, 20I8; ASF, 2018: 10; Guerrero, 2011: 265). 
Además de los subsidios, se implementaron tres políticas que delinearon el proyecto de reforma policial. En primer lugar, se puso en marcha el programa de Policía Estatal Acreditable, por medio del cual se pretendía dotarlas de una estructura básica de operación que contemplaba la creación de tres unidades de investigación, análisis táctico y operaciones. En segundo lugar, se implementó un sistema de certificación policial (con control de confianza), el cual en la práctica resultó ser un filtro y un proceso de depuración constante para todas las corporaciones del país, que tuvo profundas repercusiones en sus estados de fuerza. En tercer lugar, se impulsó el esquema de Mando Único, una estrategia por medio de la cual se pretendía que las policías municipales fueran absorbidas y controladas por los mandos de las policías estatales para integrar 32 unidades. ${ }^{4}$ Esta última medida no logró implementarse a cabalidad, pero se mantiene vigente con distintos mecanismos de coordinación entre corporaciones estatales y municipales.

Lo que interesa destacar para los fines de esta investigación es que Felipe Calderón propuso desde 2008 fortalecer las policías locales, principalmente las estatales. Los entonces gobernadores se comprometieron a implementar medidas, a crear centros de evaluación y control de confianza, a depurar sus policías y asignar más recursos. ${ }^{5}$ En concordancia con la literatura sobre las reformas (LaRose y Maddan, 2009: 339, 34I), las medidas adoptadas estaban dirigidas a establecer una línea de base, un estándar de lo que debería ser una policía estatal, un benchmarking sobre el desempeño policial, la calidad de las detenciones, eficientar el ejercicio del gasto, disminuir la corrupción y mejorar la confianza ciudadana. Calderón intentó infructuosamente que el Legislativo aprobara una ley que unificara las policías; al no conseguirlo procuró desplazarlo y negociar directamente con los gobernadores. Así, tomó la batuta para diseñar los indicadores y en sucesivos momentos trató de imponerlos o negociarlos con los gobernadores.

La reforma y sus componentes pasaron a manos de Enrique Peña Nieto en 20I2, quien continuó los esfuerzos por centralizar el manejo de las policías, impulsar un mando de policía único utilizando los subsidios federales como incentivo para lograrlo. También persistió en la conflictiva depuración de policías estatales y municipales, bajo los argumentos de incapacidad o de involucramiento con organizaciones

4 Aunque este trabajo no analiza la policía municipal y se enfoca en las actividades de los gobiernos de los estados, las nombramos porque el acuerdo de Mando Único las involucra.

5 Acuerdo Nacional por la Seguridad, la Justicia y la Legalidad, Diario Oficial de la Federación (25/08/2008). 
criminales. Igualmente mantuvo al Ejército en seguridad pública, aunque disminuyó su involucramiento en varios estados (Silva y Padilla, 2020).

Este proceso creó una arena de acción política con una dinámica conflictiva, una pugna contra las policías, los estados y los municipios bajo distintos pretextos políticos (Sabet, 2012), ${ }^{6}$ que convirtió los acuerdos en instrumento de continua negociación entre la federación y los estados que, en su turno, ha generado cambios poco consistentes, transgresiones a las normas constitucionales, a los acuerdos de coordinación y a las competencias de cada sector, además de acrecentar las burocracias locales.

Este periodo de cambios coincide con el incremento en la pluralidad política del país, con una mayor fragmentación o pulverización de las coaliciones políticas estatales (Sánchez, 2020) y, para complicar más el escenario, un número creciente de gobiernos estatales tuvo situaciones críticas como renuncias, solicitudes de licencia, acusaciones o procesos por delitos y/o pertenencia a organizaciones criminales, etc. ${ }^{7} \mathrm{Al}$ mismo tiempo, las relaciones con el poder Ejecutivo federal cambiaron, creando mayor inestabilidad, asimetrías en los apoyos financieros y resistencias en la aceptación de los programas. Las recientes detenciones en los Estados Unidos de Genaro García Luna y del general Salvador Cienfuegos, altos mandos de seguridad pública de gobiernos anteriores, muestran que el problema también ocurría en la cúpula de los regímenes políticos. Esta situación se expresa en dos formas: por una parte, la lentitud y contrariedad con la que se han puesto en operación los cambios y, por la otra, la respuesta de las organizaciones policiales que han buscado mantener o incrementar su autonomía frente a los vaivenes políticos, el descrédito y la desconfianza general.

6 Este trabajo concuerda con la idea de Sabet (2012:215-217) de que las policías municipales no tenían capacidad para enfrentar todos los problemas, pero unificarlas solo porque estaban fallando no resolvió la inseguridad.

7 Entre 2006 y 2018, 16 gobernadores han sido acusados, juzgados y procesados por diversos crímenes, desde desvío de fondos hasta ser parte de organizaciones criminales (Alvarado, 2019). Por ejemplo, el gobernador de Michoacán Fausto Vallejo renunció a su cargo el I9 de junio de 2014 por acusaciones contra su gabinete por vínculos con organizaciones criminales. El exgobernador de Tamaulipas Eugenio Hernández fue detenido en octubre de 2017 por asociación con crimen organizado, entre otras cosas. Ángel Aguirre, de Guerrero, renunció el 23 de octubre de 2014 como consecuencia de la desaparición de 43 estudiantes de Ayotzinapa, en septiembre de 2012, en Iguala, Guerrero. 
Las consecuencias de estos cambios no pueden ser más desfavorables. En varias regiones del país han ocurrido situaciones críticas de violencia y de ausencia de autoridad en materia de seguridad y de ejercicio de las leyes y el orden, por lo que la federación ha tenido que capturar y remplazar cuerpos enteros de policías municipales y estatales. Junto con estas intervenciones ocurre una larga, intermitente e inconsistente reorganización del "sector" seguridad, particularmente de las policías federales, estatales y municipales, por lo cual hoy existen más de i 66I corporaciones de seguridad pública federal (García, 2006), estatal y municipal, con modelos mixtos (mandos únicos, coordinados, policías estatales, policías o fuerzas especiales; policías de proximidad, comunitarias) que discrepan del proyecto de una policía estatal acreditable y certificada. Hay una reorganización de las funciones y de las jurisdicciones de las policías estatales, nuevas policías investigadoras y cambios o eliminación de las ministeriales.

Acorde con lo anterior, resulta indispensable un análisis que ayude a distinguir las capacidades desarrolladas por los gobiernos estatales en materia de seguridad pública durante dicho periodo. Para cumplir tal cometido proponemos un marco analítico más abarcador al de las investigaciones mencionadas, que fija la mirada en las entidades más que en la federación.

\section{Propuesta analítica}

Nos centramos en la noción de capacidad porque está asociada con el desempeño, la eficacia y la calidad de la acción en las organizaciones. Esto comprende la habilidad de formular políticas y ejecutarlas, de mantener un cuerpo civil permanente dedicado a consumar, monitorear y cumplir los programas. También está relacionada con la capacidad de ejercer las finanzas. Todo esto es parte del complejo organizacional de un Estado burocrático que administra la seguridad y el ejercicio de la coerción; incluye tanto a los actores dedicados al diseño y la implementación de las políticas como a los cuerpos de policías que las ponen en práctica.

Hay numerosas teorías sobre las policías, sus fortalezas, sus capacidades y dimensiones, sustentadas en las disciplinas sociales. Stucky (2005: I4I) menciona tres enfoques o paradigmas: el de la elección pública, el del conflicto y el organizacional. El primero trata de explicar el modo en que funcionan las organizaciones. Propone que las decisiones de reformas y en general el diseño de políticas públicas deben seguir un proceso racional y comprehensivo, en el cual el problema que se busca resolver y las metas que se plantean deben estar claramente establecidos, igual que 
las alternativas, de modo que las decisiones que tomen las autoridades sean las más eficientes. ${ }^{8}$ Este enfoque ha ido mejorándose, desde la primera formulación de políticas racionales adecuadas para los objetivos claramente planteados a los modelos críticos como el del "bote de basura".

En segundo lugar, las teorías del conflicto proponen que la fuerza policial responde a los conflictos generados por la competencia de grupos ciudadanos (sociales, de clase, raciales) con diferentes intereses y posibilidades de beneficiarse de la captación de ese bien público. Así, el control del crimen estaría asociado con la protección no solo de los ciudadanos, sino particularmente de ciertos grupos poderosos que imponen una visión dominante de los delitos. Todas las teorías toman en cuenta los delitos como un indicador de las respuestas, pero no como el único. ${ }^{9}$

En tercer lugar está la literatura que propone que el comportamiento policial se explica por los imperativos organizacionales, que a su vez expresan la relación entre la forma de organización del Estado y las policías (Wilson, 1968). ${ }^{10}$

8 Según Aberbach y Christensen (2013:4), este paradigma de decisiones racionales comprehensivas propone que las políticas deben diseñarse después de un análisis cuidadoso, que incluya un "cálculo racional" (Dahl y Lindblom, 1953), para resolver el problema, para rediseñar un sistema o implementar una política con éxito. Esto supone que tenemos una idea clara para medir cada etapa del proceso. Esta línea continuó con Simon (1957) y Cohen, March y Olsen (1972).

9 En la literatura referida hay un amplio debate sobre la relevancia de medir los avances solamente con base en los resultados en la reducción de delitos. Es metodológicamente muy difícil comprobar que efectivamente las acciones de un programa de un gobierno estatal y su policía son las que causaron la disminución de los delitos dentro de su territorio en un tiempo dado, porque están afectadas por el ambiente regional y nacional. No solo es un tema de eficacia (Llanos et al., 2018). Los expertos coinciden en que la capacidad debe medirse no solo por la reducción de los delitos, sino apoyándose en otros indicadores que muestran la multicausalidad. En nuestro trabajo, los indicadores utilizados son de desempeño y de percepción ciudadana, ya que esto centra la capacidad de las reformas para realizarse.

${ }^{10}$ Wilson (1968) vincula la política local con la estructura de organización de la policía. En la misma vertiente están Stucky (2005:143) y Wilson y Boland (1978). Pero otros proponen diferentes explicaciones. Por ejemplo, Langworthy (1986), Maguire y Uchida (2000: 533) y Koper et al. (2001:32) sugieren que el tamaño de la policía está determinado por causas internas. Otros proponen que el mejor predictor de su comportamiento es la fuerza inercial previa, medida por el número de elementos (Stucky, 2005: 14I; Brandl, Chamlin y Frank, 1995; Chamlin y Langworthy, 1996). 
En concordancia con estos enfoques organizacionales utilizados en los trabajos policiales, los estudios de políticas públicas proporcionan suficientes elementos para explicar los procesos de cambio y reformas. Además, nos permiten encuadrar la pregunta de por qué ha sido tan difícil poner en práctica las reformas y por qué parecen no funcionar, pues no basta con demostrar que no sirven, es necesario entender las razones del fracaso y sus consecuencias. El primer modelo supone un sistema de jerarquías y un procedimiento de negociación de las decisiones entre los actores. Como lo mostraremos en el trabajo, las reformas policiales mexicanas carecen de dicha racionalidad económica, administrativa e instrumental, entre otras cosas por la vaguedad de las metas, las dificultades de negociación y la heterogeneidad de posturas entre los participantes.

En respuesta a los problemas del primer enfoque surgió una interpretación llamada "bote de basura" (Garbage Can Model de Cohen March y Olsen, 1972), que ofrece una explicación alternativa acerca de cómo funcionan las organizaciones, sobre todo en dos componentes: el proceso que lleva a las decisiones de reforma y el proceso de su implementación ( $y$ su negociación para aprobarlas y evitar bloqueos). Plantean que las preferencias no siempre son claras y consistentes, de manera que pareciera haber soluciones buscando problemas y no viceversa, porque: “... [las] preferencias [se manifiestan] a través de las acciones, antes que las acciones a través de las preferencias" (Cohen, March y Olsen, 1972: 249. También Aberbach y Christensen, 2013:3).

Los procesos son confusos, a veces tienen errores y la participación es "fluctuante", ya que no todos invierten el mismo tiempo, esfuerzo y atención; a menudo cambian de preferencias o, incluso, de personas a cargo, que naturalmente tienen posturas y capacidades diferentes (Cohen, March y Olsen, 1972: 250). Esto lleva a que las decisiones de reforma no siempre cuenten con un claro compromiso y que la implementación esté sujeta a fluctuaciones y equívocos. En este sentido, es preciso explicar lo que ocurre cuando las metas no son claras ni han sido consensuadas con compromisos firmes en favor de la reforma, o cuando los autores no tienen la autoridad para hacerlas cumplir, como sería el caso de la Presidencia frente a los gobernadores. Otro problema es el mecanismo político administrativo para implementar los cambios. ${ }^{11}$ Además, es necesario tomar en cuenta otros aspectos, como

${ }^{11}$ Este modelo crítico explica parcialmente las reformas policiales. Parte de un statu quo de no hacer nada en la mayoría de las entidades (excepto el ex Distrito Federal y algunos municipios que antes de 2008 implantaron policías comunitarias o metropolitanas, con modelos de seguridad ciudadana, en Chihuahua, Guerrero o Jalisco), hacia un acuerdo 
la capacidad de liderazgo, capacidad gerencial de los gobernadores, por ejemplo, para fijar la agenda (Kingdon y Sotano, 1984), temas normativos, políticos, contextuales y de temporalidad, pues hay un periodo de oportunidad limitado para realizar las reformas, que también constriñe la posibilidad de continuarlas y de emplear los recursos. Esto requiere considerar acertadamente los temas de mando y jerarquía dentro del régimen político (Dahl y Lindblom, 1953).

Frente a los debates y algunas insuficiencias de los primeros paradigmas surgió el tercer grupo de literatura que pone énfasis en los incentivos institucionales y la gobernanza (Ostrom, 2000, 2005; Polidano, 2000), la cual nos sirve para integrar los modelos precedentes porque propone que para explicar el comportamiento policial debemos incluir elementos de la propia organización, como también del contexto (que llama ambiente), de la cultura política local y la forma de gobierno. Este paradigma considera los arreglos institucionales y normativos del régimen político mexicano, las reglas de actuación en materia de seguridad, las condiciones políticas e institucionales con las que trabajan las policías estatales (los agentes) y sus jefes, los gobernadores.

Para incorporar estos modelos en nuestro análisis es necesario analizar ( $y$ especificar) el tipo de régimen político, que es el presidencial: un sistema que tiende a procesar de manera "lenta" las políticas, cuando no las paraliza o las obstruye, con momentos excepcionales de avances (Ostrom, 2005: 10), debido entre otras cosas a las jerarquías entre distintos órdenes de gobierno y a las diferencias entre los intereses y preferencias de los involucrados. Los actores con capacidad de influencia local son el presidente y el Sistema Nacional de Seguridad Pública (SNSP); este predominio del Ejecutivo se reproduce en las entidades federativas con diferente grado de intensidad con los gobernadores, en ocasiones los congresos locales, algunos grupos de poder, las policías y los distintos grupos criminales que operan. Es un juego de enorme competencia e inestabilidad.

Tomando en cuenta lo anterior, para este trabajo entenderemos por capacidad institucional el conjunto de componentes del Estado para ejercer el poder, junto con aquellos recursos destinados para ejecutar las políticas públicas. Respecto de la capacidad del sector público, incluimos los siguientes componentes: aspectos

colectivo, que opera cambios con metas vagas y cambiantes y con técnicas poco discutidas (entrenamiento, certificación, combate a la corrupción). Los procedimientos son confusos (las reglas de reforma hechas por la federación son inadecuadas, inaceptables o imposibles de realizar). En medio de esto los gobernadores buscan beneficiarse, además de intentar mostrar a sus ciudadanos que tienen control sobre la criminalidad. 
normativos, ${ }^{12}$ administrativos y políticos, esto es, la capacidad de la autoridad para crear políticas, ponerlas en marcha con recursos y operarlas eficientemente, es decir, hacerlas cumplir. Tanto Polidano (2000: 805) como Ostrom consideran que estas capacidades son condiciones facilitadoras. Y siguiendo la propuesta de Ostrom, la capacidad incluye la existencia de ciertos programas de reforma (leyes, convenios) y otros indicadores de desempeño (unificación, certificación, detenciones, actuación legal y legítima), de ejercicio y monitoreo del gasto. Así, las policías estatales y sus dirigentes serán considerados parte de la maquinaria permanente del gobierno para definir y ejecutar la política en seguridad. ${ }^{13}$

Por lo que se refiere a la actuación de las policías, proponemos que hay un conjunto de condiciones bajo las cuales operan en lo que Ostrom llama la "arena de acción" (2005ः 6, 15). Comprenden los arreglos institucionales de los sistemas de seguridad federal y de las entidades federativas. A estos aspectos agregamos un argumento sobre las policías, que hace referencia a su capacidad de aislarse y crear autonomía, porque la policía se organiza primero que nada para garantizar su sobrevivencia en un ambiente político, social y criminal adverso, y luego cumplir sus tareas, como combatir los delitos (Alvarado, 2012) y tratar de salir del paso (Lindblom, 1959: 8I).

¿Cómo se comportan los actores dentro del juego de la seguridad $?^{14} \mathrm{La}$ conducta de los políticos y de las policías es un problema de acción colectiva (Olson, I965, en Ostrom, 2005: 24). En algunos casos las estrategias son cooperativas, con juegos de sumas positivas, mientras que en otros son de conflicto o juegos de suma cero. Cada actor tiene preferencias y un horizonte de opciones relacionadas con sus ciudadanos, con el gobierno nacional y con el crimen. El juego incluye varias opciones, desde el compromiso completo con una política propia o alguna propuesta del Ejecutivo, hasta la oposición y resistencia a las políticas federales, el enfrentamiento

12 En un contexto en el que se acostumbra que las normas en uso no siempre correspondan con las formales, o que ambas operen simultáneamente.

${ }^{13}$ Nuestro modelo cubre también los criterios de capacidad policial propuestos por la Organización de las Naciones Unidas: I) profesionalismo e integridad (incluye comando y poder); 2) administración (recursos humanos y financieros); 3) marco legal y de políticas; 4) mecanismos de rendición de cuentas y 5 ) involucramiento ciudadano (UN-POLI$\mathrm{CE}, 2017: 6$ ). Esto es sinónimo de construcción institucional-estatal (ONU, 2017: 36).

${ }^{14}$ Aquí de nueva cuenta es pertinente considerar algunos aspectos del modelo "bote de basura", ya que los actores no tienen preferencias fijas, cambian con el tiempo. Hay ajustes en los costos del programa y las alternativas para llevarlo a cabo (Aberbach y Christensen, 2013: 7-8). 
o la complicidad criminal, ${ }^{15}$ pasando por distintas respuestas que cubren opciones de acuerdos parciales entre el presidente y los gobernadores, acciones oportunistas $y$ de free riding.

Así, por ejemplo, para Sabet (2012) las reglas informales del presidencialismo y clientelismo se combinan con las normas constitucionales para producir incentivos que sistemáticamente han socavado la profesionalización de la policía, pues entorpecen el desarrollo de un sistema de promoción basado en méritos, la institucionalización de reformas y la rendición horizontal de cuentas. ${ }^{16}$

Para los gobernadores, las alternativas y los dilemas -cambiantes a lo largo del tiempo- serían: cooperar con recursos monetarios y humanos, o no hacerlo y esperar a que otros lo hagan y paguen el costo. Sin embargo, aquí hay una paradoja: a pesar de que la estrategia de utilizar los apoyos federales sin pagar los costos es aparentemente la más barata, los gobiernos tienen un gasto considerable, tanto que en ocasiones el presupuesto local es mayor que el subsidio federal. Todavía más, a pesar de que no es poco común un subejercicio del subsidio federal, a nivel local los gobiernos suelen gastar más de lo presupuestado (Sánchez, 2020:157, 158), lo cual es indicador de malas prácticas y baja institucionalidad.

En este sentido, de acuerdo con la literatura (Ostrom, 2005; Wilson, 2005; Stucky, 2005), sería de esperarse, en un contexto de cambios e innovación, que los gobernadores y sus policías interesados en construir capacidades invirtieran más recursos propios, se asociaran de manera condicional o total con la federación cumpliendo con sus directrices y procurando mejorar la satisfacción ciudadana y sus policías (dinero, equipo, entrenamiento, más empleos). ${ }^{17}$

15 Estas conductas han sido reiteradas a lo largo de una década. Véanse las acusaciones del secretario de Seguridad, Durazo, por la ausencia de varios gobernadores en las reuniones del SNSP (Solís, 2019).

${ }^{16}$ De acuerdo con Sabet (2012) el propósito de los gobernadores de distinguirse de su predecesor produce un efecto de péndulo: una administración se enfoca en mejorar la calidad de la policía y otra intenta incrementar el número de elementos; una centraliza el mando y otra descentraliza o desarrolla un estilo militar de fuerza y la otra aboga por una fuerza orientada a la comunidad. Así ocurrió en Jalisco entre los gobiernos de Aristóteles Sandoval y Enrique Alfaro.

${ }^{17}$ Por su parte, el recurso decisivo con que cuentan las policías (el agente) para aceptar o rechazar un cambio es su control del ejercicio de la coerción. Los políticos saben que es mejor no permitir su veto, simulación o enfrentamiento. Además, sería de esperarse, si las policías son propensas al riesgo, que tiendan a enfrentar activamente los retos de la delincuencia; pero en caso contrario tenderán a reducirlos y a buscar mecanismos de 
Dos temas adicionales deben ser tomados en cuenta en las reformas policiales. Autores como Christensen et al. (2017) y Sirnes y Lægreid (2019) han analizado las reformas de las policías noruegas en años recientes y encontraron que la perspectiva estructural e institucional en los procesos de reforma no son suficientes para explicarlas. Sus investigaciones coinciden en que factores culturales, la configuración de la agenda, el cambio de atención y los factores situacionales, así como el path dependency, son más importantes en la reforma. Por su parte Sirnes y Lægreid (2019) subrayan la falta de colaboración política para la instauración de medidas de seguridad, porque hay guerras territoriales entre áreas ministeriales con respecto a competencias y responsabilidades. Las organizaciones prefieren coordinar que ser coordinadas y en caso de no lograr imponerse desarrollan un esquema de coordinación negativa.

Para el caso de México es importante tomar en cuenta que el path dependency ha sido depender de la federación debido al centralismo y al escaso desarrollo de las instituciones locales; sin embargo, las cuestiones contextuales y coyunturales que han desatado la violencia, así como la tendencia a la politización de los temas de seguridad han propiciado que algunos gobiernos estatales se vuelvan más protagónicos.

\section{Hipótesis}

De acuerdo con la literatura, no hay una única dimensión que explique la creación de capacidades, pues estas son resultado de muchos factores. En este sentido, se proponen tres hipótesis que los agrupan:

Hipótesis I: sobre el gasto estatal en seguridad. Hay una relación positiva entre el aumento del gasto y la creación de capacidad. Las preferencias de los actores por construir mejores políticas se revelan en la formulación y el ejercicio del gasto público local y, como complemento, el aprovechamiento del subsidio federal. Cuando una entidad conviene el apoyo federal y acepta las reglas y los programas, está adquiriendo capacidades administrativas y técnicas para el ejercicio de los recursos, ya sea por la vía de crearlas o de importar las federales. Sería de esperarse, si la generación de capacidades está determinada por las preferencias de los gobernadores, que un aumento claro del gasto local exprese su preferencia por estimularlas. La acción está

protección (no hacer nada, acomodarse, involucrarse con organizaciones ilícitas, lo cual además genera rentas). Todo esto podría llevar no solo al colapso de una reforma, sino a medidas de acomodo, simulación y otros resultados indeseables de la política pública. 
positivamente asociada con las demandas ciudadanas por tener mejor seguridad. En algunas entidades el gasto se relaciona con una burocracia capaz de definir un programa, establecer metas y ejecutarlas. Es de esperarse también que estas iniciativas cuenten con el apoyo de las policías, aun si es condicionado.

Por lo tanto, podemos hipotetizar que un mayor gasto local generaría mayor capacidad, mayor interés político-institucional y una preferencia por responder a la demanda ciudadana de mejorar la seguridad y resolver la criminalidad. Ese interés político institucional también se debería traducir en un estado de fuerza suficiente y en una mayor percepción de seguridad por parte de los ciudadanos.

Hipótesis 2: sobre el papel de los gobernadores en el fortalecimiento institucional y las relaciones entre los diferentes órdenes de gobierno, derivados de los enfoques organizacionales sobre el liderazgo, la gerencia, la jerarquía y las capacidades de negociación. Esta hipótesis postula que los gobernadores tienen la opción de seguir los directrices de la federación para fortalecer sus instituciones (capacitar a su policía, entre otras cosas), mientras que la opción contraria es decidir no fortalecer su policía y esperar a que la federación resuelva el problema mediante la intervención de la policía federal y las fuerzas armadas (en otras palabras, ser un free rider y esperar el rescate).

Hay un segundo componente de esta hipótesis sobre fortaleza de los gobernadores, que consiste en su capacidad de implantar el acuerdo de mando único. Los gobernadores más capaces serían aquellos que lograran mayor cantidad de acuerdos con sus municipios. El paradigma organizacional propone que los actores de la jerarquía del sistema pueden hacer cumplir las propuestas cuando establecen medidas eficaces de negociación con los municipios. ${ }^{18}$

La tercera hipótesis se refiere al desarrollo de capacidades policiales específicas, que puede medirse de diferentes formas. Avanzamos en una propuesta que mejora los indicadores tradicionales de actuación, como las detenciones, el tiempo de respuesta, las multas, entre otros. ${ }^{19}$ Nos interesa examinar indicadores integrales que reflejen algunos cambios en las prácticas policiales, orientados al respeto a la

18 Es necesario reiterar que el trabajo no analiza los municipios, sino solamente las actividades de los gobiernos de los estados. El mando único es un acuerdo paralelo que explica la capacidad de liderazgo y gestión de los gobernadores.

19 Todas las policías acatan las medidas, pero eso no indica que cumplan sus objetivos ni que respeten la ley. Los indicadores tradicionales no rinden cuentas a la comunidad en temas de control de la corrupción, impunidad, abusos de autoridad, tortura, desorden público ni uso ilegítimo de la fuerza. Ignoran la reducción del miedo y la satisfacción ciudadana. 
ley y al "debido proceso", tales como la calidad de las detenciones, la reducción de la corrupción y la rendición de cuentas a la ciudadanía, esto es, a la construcción de confianza. Una policía que acepta cambiar y fortalecerse tenderá a actuar con apego a protocolos basados en derechos humanos para las detenciones, puestas a disposición, investigación, profesionalización, certificación y evaluación.

Buscamos medir la capacidad de los gobiernos para construir policías profesionales, entendidas como aquellas con un estado de fuerza suficiente, cercano al óptimo de la onu (Muedano, 20I8; ONU, 20I7): una policía que actúe con apego al estado de derecho y que cumpla las expectativas de la ciudadanía. Así que mientras más alto aparezcan las policías en estos indicadores, mayor capacidad muestran los gobiernos para construir policías profesionales.

Es importante precisar que cada hipótesis tiene diferentes variables y en cada una habrá un grupo de estados que las cumpla y otros que no. Sin embargo, más allá de las tendencias que se observen en cada variable, la intención principal del artículo es presentar una propuesta de medición que permita conjuntarlas y mostrar el avance de las capacidades de los gobiernos y las policías estatales de manera comparativa, así como clasificar las entidades de acuerdo con su grado de avance. Veamos la metodología.

\section{Método}

Para analizar la capacidad para desplegar una política de seguridad pública por parte de los gobiernos estatales creamos una base de datos construyendo siete variables ( $y$ nueve indicadores) de distintas fuentes que son las más adecuadas o proxys de los procesos que se discutieron en los apartados anteriores ${ }^{20}$ Cada variable está asociada

Las fuentes de la base de datos son las siguientes: el Secretariado Ejecutivo del Sistema Nacional de Seguridad Pública proporcionó los datos correspondientes al estado de fuerza de las policías. El gasto en seguridad de los estados proviene de los presupuestos de egresos de los 32 estados publicados en sus respectivos periódicos oficiales de los años seleccionados. Se consideraron variables de dos encuestas del Instituto $\mathrm{Na}$ cional de Geografía y Estadística (INEGI), de la Encuesta Nacional de Victimización y Percepción de la Seguridad Pública (Envipe) la cual se aplica a ciudadanos mexicanos. También se tomaron algunas variables de la Encuesta Nacional de Población Privada de la Libertad (ENPOL), que se aplica a personas que están en la cárcel. Finalmente se considero el Índice de Desarrollo Policial creado por la asociación civil Causa en común, Indepol 2017, que puede consultarse en: <https://causaencomun.org.mx/beta/ 
con alguna de las hipótesis planteadas en el apartado anterior. Pero hay que hacer énfasis en que es el conjunto de las variables el que da cuenta de las capacidades estatales. El índice se construyó mediante análisis factorial, utilizando la técnica de factores principales, el cual nos permite clasificar los estados tomando en cuenta cada una de las dimensiones de las que informan las variables agrupadas para cada una de las hipótesis.

De acuerdo con Urbina y Bárcena (2019:53), el método de factores o componentes principales parte de un modelo hipotético en el que se asume que un determinado número de factores, mucho más pequeño que el número de variables en escrutinio, son responsables de las correlaciones observadas. En ese sentido, asumimos que todas las variables propuestas informan de las diferentes dimensiones de la capacidad de los estados para desplegar una política de seguridad pública. No obstante, el peso de cada una de esas dimensiones no es el mismo. En consecuencia, la técnica de análisis factorial por factores principales permitirá ponderar cada uno de los componentes del índice de acuerdo con las correlaciones existentes entre variables, y de esta manera obtendremos una medida más precisa y confiable.

\section{Cuadro i:}

Hipótesis, variables e indicadores para el estudio de las capacidades

en seguridad pública de los gobiernos estatales.

\begin{tabular}{lll}
\hline Hipótesis & Variables & Indicadores \\
\hline & $\begin{array}{l}\text { Gasto en } \\
\text { seguridad }\end{array}$ & $\begin{array}{l}\text { \% del gasto en seguridad respecto del gasto } \\
\text { total estatal para 20I6 (SENSPSP). }\end{array}$ \\
\cline { 2 - 3 } $\begin{array}{l}\text { Gasto en seguridad } \\
\text { (autonomía) }\end{array}$ & $\begin{array}{l}\text { Percepción } \\
\text { de seguridad }\end{array}$ & $\begin{array}{l}\text { \% de personas que consideran su entidad segura } \\
\text { y muy segura (ENVIPE, 20I6). }\end{array}$ \\
\cline { 2 - 3 } & $\begin{array}{l}\text { Estado de } \\
\text { fuerza }\end{array}$ & $\begin{array}{l}\text { Tasa de policía por mil habitantes para 20I6 } \\
\text { (SESNSP). De acuerdo con la onu, el óptimo } \\
\text { es I.8 }\end{array}$ \\
\hline $\begin{array}{l}\text { Relación gobierno } \\
\text { federal-gobierno } \\
\text { estatal }\end{array}$ & $\begin{array}{l}\text { Cumplimiento } \\
\text { de acuerdos }\end{array}$ & $\begin{array}{l}\text { Calificación otorgada a la entidad de acuerdo } \\
\text { con el Índice de Desarrollo Policial de Causa } \\
\text { en Común (Indepol 20I7).* }\end{array}$ \\
\hline
\end{tabular}

indepol-indice-de-desarrollo-policial/>. 


\begin{tabular}{|c|c|c|}
\hline Hipótesis & Variables & Indicadores \\
\hline \multirow{5}{*}{$\begin{array}{l}\text { Desarrollo de } \\
\text { policías profesionales }\end{array}$} & \multirow{3}{*}{$\begin{array}{l}\text { Desempeño } \\
\text { policial }\end{array}$} & $\begin{array}{l}\% \text { de detenidos en flagrancia o con orden de } \\
\text { aprehensión (ENPOL-INEGI, 20I6) }\end{array}$ \\
\hline & & $\begin{array}{l}\text { \% de detenidos que manifestaron que la policía } \\
\text { se identificó y explicó la razón del arresto } \\
\text { (ENPOL-INEGI,20I6) }\end{array}$ \\
\hline & & $\begin{array}{l}\text { \% de personas que consideran el desempeño } \\
\text { de la policía estatal como bueno y muy bueno } \\
\text { (ENVIPE -INEGI, 20I6) }\end{array}$ \\
\hline & Corrupción & $\begin{array}{l}\% \text { de personas que consideran que su policía } \\
\text { es poco y nada corrupta (ENVIPE-INEGI, 20I6) }\end{array}$ \\
\hline & Confianza & $\begin{array}{l}\% \text { de personas que confían mucho y algo en su } \\
\text { policía (ENVIPE-INEGI, 20I6) }\end{array}$ \\
\hline
\end{tabular}

Fuente: elaboración de los autores con base en Indepol, 2017.

*El índice de desarrollo policial Indepol verifica cómo están las policías estatales y federal en función de lo que establece la ley respecto al desarrollo policial.

Los indicadores con los cuales se trabajó permiten realizar una medición para 20ı6, debido a la dificultad de construir series de tiempo para cada uno de los indicadores. Tomando en cuenta lo expuesto, el análisis de las capacidades estatales para ese año tiene ventajas y desventajas. Por un lado, permite comparar a los 32 estados casi una década después de que se introdujeran las reformas a las policías estatales. El hecho de que Peña Nieto no haya realizado grandes cambios a los planes de reforma vuelve menos problemática esta medición. Por otro lado, analizar tan solo un año no permite evaluar fluctuaciones anuales en los indicadores que posibiliten una discusión mucho más compleja. No obstante, como se ha venido argumentando, esta propuesta representa un primer paso para comenzar un análisis comparativo a nivel estatal de las reformas que tome en consideración la percepción de la ciudadanía.

\section{Resultados}

Por cuestiones de extensión, nos concentraremos en los resultados que integran el índice en el cuadro. Para cada indicador se calculó el promedio nacional y de esta forma fue posible identificar los estados que estaban por debajo y por arriba de la media, y evaluar su relación con las hipótesis planteadas. Para cada indicador se destacó la entidad que obtuvo el valor más alto (con negritas y subrayado), la que 
obtuvo el valor más bajo (fondo negro y letras blancas) y aquellas cuyos valores estuvieron por encima de la media (recuadro en gris), es decir, todas las que obtuvieron un resultado que permite hablar del desarrollo de esa capacidad. Los resultados fueron los siguientes:

\section{Cuadro 2}

Concentrado de los resultados por indicador

\begin{tabular}{|c|c|c|c|c|c|c|c|c|c|}
\hline Entidad & 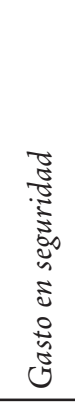 & 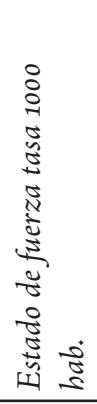 & 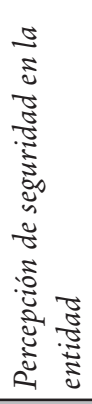 & 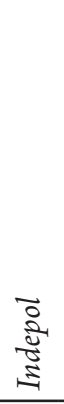 & 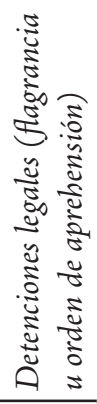 & 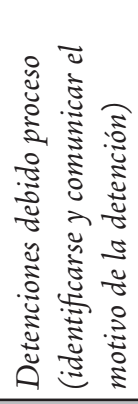 & 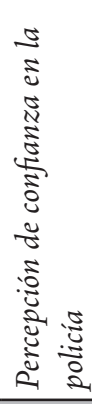 & 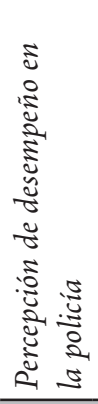 & 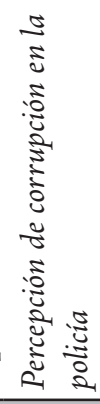 \\
\hline Aguascalientes & 2.93 & 0.54 & 3 & 49 & 50 & 34 & 65 & 70 & $4 \mathrm{I}$ \\
\hline Baja California & I.27 & 0.47 & 2 & 78 & 38 & 26 & 6I & 44 & 26 \\
\hline Baja California Sur & 3.26 & 0.94 & 2 & 44 & 52 & 40 & 62 & 57 & 37 \\
\hline Campeche & 2.86 & I.5I & 3 & 62 & 55 & 29 & 66 & 59 & 32 \\
\hline Chiapas & 2.30 & $\mathrm{I} .42$ & 3 & 50 & 36 & 23 & $6 \mathrm{I}$ & 54 & 33 \\
\hline Chihuahua & 2.23 & $\mathrm{I} .34$ & 2 & 55 & 69 & 50 & 62 & $5 \mathrm{I}$ & 29 \\
\hline Ciudad de México & 9.25 & $\underline{10.02}$ & 2 & 69 & 65 & 46 & 36 & 35 & I4 \\
\hline Coahuila & 2.40 & 0.63 & 3 & 50 & 33 & 26 & 64 & $6 \mathrm{I}$ & 35 \\
\hline Colima & 3.79 & $\mathrm{I}+24$ & $\underline{16}$ & 62 & 62 & 29 & $5 \mathrm{I}$ & 60 & 39 \\
\hline Durango & 1.85 & 0.56 & 3 & 57 & $4 \mathrm{I}$ & 38 & 48 & 49 & 33 \\
\hline Estado de México & 3.73 & I.19 & I & 48 & $5 \mathrm{I}$ & 27 & 46 & 39 & 17 \\
\hline Guanajuato & 2.68 & 0.40 & 2 & 59 & 37 & 25 & 63 & 67 & 39 \\
\hline Guerrero & $3.8 \mathrm{I}$ & 1.69 & I & 68 & 57 & 30 & 57 & 50 & 29 \\
\hline Hidalgo & 3.18 & $\mathrm{I} . \mathrm{O} 3$ & 3 & 55 & 54 & 26 & 65 & 62 & 30 \\
\hline Jalisco & 5.08 & 0.82 & 2 & 64 & 62 & 32 & 64 & 67 & 35 \\
\hline Michoacán & 3.77 & 1.06 & 2 & 67 & 54 & 26 & 59 & 54 & 33 \\
\hline Morelos & 3.01 & 1.20 & I & 77 & 39 & 19 & 45 & 36 & I8 \\
\hline Nayarit & 3.91 & I.I7 & 3 & 44 & 45 & 27 & 65 & 67 & 37 \\
\hline Nuevo León & 4.39 & I.09 & 2 & 63 & $6 \mathrm{I}$ & 29 & 72 & 72 & 49 \\
\hline
\end{tabular}




\begin{tabular}{|c|c|c|c|c|c|c|c|c|c|}
\hline Oaxaca & 4.19 & I.73 & 2 & 48 & 57 & 33 & 67 & 56 & 26 \\
\hline Puebla & 3.29 & 1.15 & 2 & 75 & 68 & 35 & 60 & 50 & 28 \\
\hline Querétaro & $\mathrm{I} .7 \mathrm{I}$ & 0.46 & 3 & 63 & 64 & 34 & 66 & 62 & 32 \\
\hline Quintana Roo & I.92 & $\mathrm{I} .07$ & 2 & 54 & 67 & 38 & 57 & 50 & 21 \\
\hline San Luis Potosí & $3.7 \mathrm{I}$ & $\mathrm{I} .33$ & 3 & 64 & 55 & 34 & 52 & 52 & 29 \\
\hline Sinaloa & 6.74 & $0.7 \mathrm{I}$ & 3 & 58 & 72 & 52 & 58 & 57 & 28 \\
\hline Sonora & 3.85 & $0.4 \mathrm{I}$ & 2 & 45 & 55 & 35 & 60 & 56 & 34 \\
\hline Tabasco & 4.35 & 2.23 & 2 & 65 & $5 \mathrm{I}$ & 35 & 54 & 45 & 22 \\
\hline Tamaulipas & 4.29 & 1.15 & 2 & 62 & $6 \mathrm{I}$ & 37 & 54 & 52 & 37 \\
\hline Tlaxcala & 3.75 & $\mathrm{I} .27$ & 3 & 46 & 70 & 27 & 59 & 52 & 23 \\
\hline Veracruz & 7.85 & I.47 & 2 & 69 & 37 & $2 I$ & 47 & 44 & 30 \\
\hline Yucatán & 3.60 & 2.05 & 4 & 55 & 46 & 32 & 74 & 72 & 35 \\
\hline Zacatecas & 2.46 & 0.78 & 2 & 50 & 64 & 39 & 65 & $6 \mathrm{I}$ & 42 \\
\hline
\end{tabular}

Fuente: Elaboración por parte de los autores con base en las fuentes señaladas para cada indicador en el cuadro $\mathrm{I}$.

\section{Descripción sistemática de los resultados para las variables y los indicadores}

Comencemos con el gasto en seguridad. En promedio los estados destinan 3.65\% del presupuesto disponible anualmente a la seguridad pública. El estado con mayor gasto fue la Ciudad de México con 9.25\% (aunque debemos tomar en cuenta que debido a su importancia por ser capital y a su densidad poblacional, su presupuesto y estado de fuerza son mucho mayores que el resto). En segundo lugar se ubicó Veracruz, con $7.8 \%$, y posteriormente otras I4 entidades se colocaron por encima de la media. En el polo opuesto figuran Baja California (I.27\%), Querétaro (I.7I\%) y Durango (I.85\%), con el menor gasto destinado a seguridad.

Para complementar la importancia del gasto, mostramos su despliegue en el periodo en que ocurrió la reforma. 


\section{GrÁFICA I}

Evolución porcentual del gasto en seguridad estatal, 2006, 2009, 2016

Estados que disminuyen el gasto y estados que lo incrementan

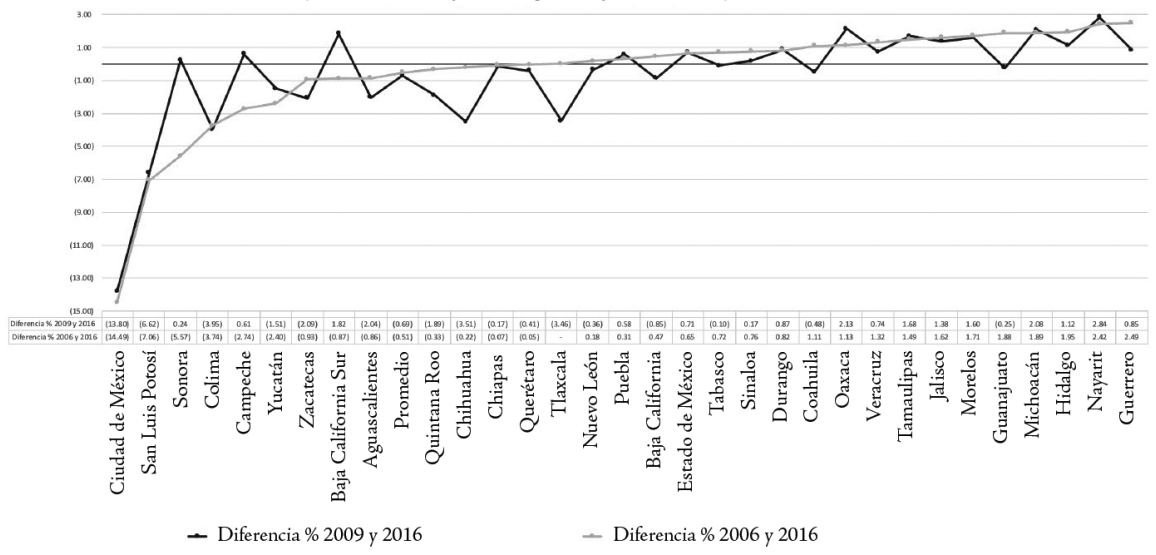

Fuente: Elaboración propia con base en los presupuestos de egresos publicados en los 32 periódicos oficiales de cada entidad.

La gráfica muestra la evolución relativa del presupuesto estatal dedicado a seguridad, como proporción del presupuesto total de cada entidad. Como sería de esperarse, algunos estados incrementaron su gasto mientras que otros lo disminuyeron en los dos periodos. El primer momento es 2006 -antes del inicio de las reformas policiales- y llega hasta 2016; el segundo se limita al lapso 2009-2016 y está relacionado con el acuerdo nacional de 2008 en el que los estados pactaron el incremento de recursos. Diez entidades redujeron su gasto porcentual y el resto lo aumentaron, pero ya lo venían incrementado desde 2006. Además, el saldo promedio del periodo es negativo. Esto muestra que las entidades federativas respondieron de manera diferente a las propuestas de reforma.

Con respecto al estado de fuerza, las policías muestran déficits importantes (tercera columna de izquierda a derecha del cuadro 2). El promedio nacional es de I.3 policías por cada mil habitantes, y de acuerdo con la onU tendría que ser de I.8. Una de las posibles causas de estados de fuerza reducidos son los exámenes de control de confianza, los cuales propiciaron filtros más estrictos de ingreso a las corporaciones y una depuración constante de los activos, producto de los reprobados en dicha prueba. La entidad con el estado de fuerza más grande es la Ciudad de México con una tasa de diez policías por cada mil habitantes. La capital del país se muestra consistente en cuanto a gasto y estado de fuerza; sin embargo, ya se ha 
mencionado que resulta un caso atípico. En segundo lugar se ubicó Tabasco con 2.23, y enseguida Yucatán con una tasa de 2.05 policías por cada mil habitantes. La entidad con la tasa más baja fue Guanajuato con apenas 0.40 policías por cada millar de habitantes. El cuadro 3 complementa la visión del estado de fuerza en las entidades.

\section{Cuadro 3.}

Clasificación estatal basada en el estado de fuerza promedio (2006-2019)

\begin{tabular}{|c|c|c|}
\hline & Policias & Grupo \\
\hline Ciudad de México & 95499 & \multirow{3}{*}{ sobresalientes $(3$} \\
\hline Estado de México & 34556 & \\
\hline Veracruz & I4 II3 & \\
\hline Puebla & 8540 & \multirow{11}{*}{ grandes (11) } \\
\hline Chiapas & 7031 & \\
\hline Oaxaca & $668 \mathrm{I}$ & \\
\hline Jalisco & 6596 & \\
\hline Nuevo León & 6246 & \\
\hline Guerrero & 5823 & \\
\hline Tabasco & 5635 & \\
\hline Michoacán & 5032 & \\
\hline Chihuahua & 4875 & \\
\hline Yucatán & 4273 & \\
\hline San Luis Potosí & 4030 & \\
\hline Hidalgo & 3746 & \multirow{10}{*}{ medianas (I2) } \\
\hline Tamaulipas & $3 \mathrm{I} 5 \mathrm{I}$ & \\
\hline Morelos & 2533 & \\
\hline Tlaxcala & 2234 & \\
\hline Guanajuato & 2183 & \\
\hline Coahuila & 2047 & \\
\hline Sinaloa & 2041 & \\
\hline Quintana Roo & I 743 & \\
\hline Baja California & 1613 & \\
\hline Campeche & I $28 \mathrm{I}$ & \\
\hline
\end{tabular}




\begin{tabular}{lcc}
\hline & Policias & Grupo \\
\hline Nayarit & I 268 & \\
\hline Sonora & I 203 & \\
\hline Zacatecas & 998 & \\
\hline Colima & 948 & \\
\hline Querétaro & 843 & \\
\hline Durango & 727 & \\
\hline Aguascalientes & 723 & \\
\hline Baja California Sur & 493 &
\end{tabular}

Fuente: organización de los autores con base en información proporcionada por el SESNSP, 2016.

Continuamos con el análisis de los indicadores de capacidad. La percepción de inseguridad (cuarta columna del cuadro 2) es un indicador que refleja una profunda inconformidad por parte de los ciudadanos con respecto al trabajo de las autoridades. La media nacional se ubica en $3 \%$, es decir, apenas $3 \%$ de los mexicanos considera que vive seguro. Colima aparece como la entidad con el porcentaje más alto de percepción positiva de seguridad con $16 \%$, muy por encima del resto del país. En contraparte figura el Estado de México con I\% de la población que afirma sentirse segura.

Tomando en cuenta las tres variables descritas es posible afirmar que ninguna entidad obtuvo una calificación óptima, es decir, por encima de la media en los tres rubros: 12 entidades obtuvieron la mejor calificación en dos de las tres variables analizadas (Chiapas, Ciudad de México, Colima, Guerrero, Nayarit, Oaxaca, Tlaxcala, Yucatán, San Luis Potosí, Tabasco y Veracruz). Acorde con estos resultados es posible afirmar que un tercio de los estados mostraron en 2016 haber tenido interés en resolver la inseguridad de su entidad. El resto fueron inconsistentes en al menos dos de las tres variables propuestas.

La segunda hipótesis es la que considera la relación entre el gobierno federal y el gobierno estatal. En términos más precisos se refiere al cumplimiento de las directrices establecidos por el Sistema Nacional de Seguridad Pública. ${ }^{21}$ Para medir ese proceso utilizamos el Índice de Desarrollo Policial (Indepol, quinta columna del cuadro 2), el cual es una iniciativa impulsada desde la sociedad civil que busca

${ }^{21}$ Otra forma de expresar el objetivo era establecer un estándar básico para medir el estado de cada una de las 32 entidades y definir una línea de base de su desarrollo (benchmarking). 
analizar el grado de cumplimiento de las directrices federales en cinco rubros: carrera policial, profesionalización, régimen disciplinario, seguridad social y certificación. El indicador corresponde a la calificación obtenida por la entidad de acuerdo con el Indepol. ${ }^{22}$ Fue el único indicador utilizado para esta dimensión de análisis debido a la falta de un reporte sistemático realizado por el Secretariado Ejecutivo del Sistema Nacional de Seguridad Pública. ${ }^{23}$

Los resultados apuntan a que, en promedio, las entidades cumplen en $58 \%$ los lineamientos del SNSP. El estado con la calificación más alta fue Baja California con 78 puntos, mientras que las entidades con el cumplimiento más bajo fueron Nayarit y Baja California Sur empatadas con 44 puntos. I7 estados se ubicaron por encima de la media, lo que quiere decir que casi dos tercios de los gobiernos estatales cumplieron con al menos $58 \%$ de los compromisos pactados con la federación en 2016. En términos generales, la federación cuenta con mecanismos débiles para dirigir el fortalecimiento de las policías. La voluntad de los estados por seguir los lineamientos existe, pero también tienen que responder a problemáticas particulares de su región.

La tercera hipótesis toma en cuenta cómo los gobiernos, a través de sus policías, han ejecutado una política de seguridad apegada a derecho, respetuosa de los derechos humanos (columnas 6 y 7 del cuadro 2). Para analizar esta hipótesis se tomaron en cuenta cinco indicadores, dos de ellos referentes al proceso de detención (que estuviera justificada legalmente y si los policías se identificaron y expusieron al detenido la razón del arresto). El promedio nacional para ambos indicadores fue de $52 \%$ y $31 \%$, respectivamente. Los otros tres indicadores (columnas 8 a IO) corresponden a la percepción ciudadana en términos de la confianza que tienen en la policía, la opinión que asumen de su desempeño y si la consideran poco o nada corrupta. Los promedios nacionales para los tres indicadores fueron $58 \%, 55 \%$ y $31 \%$, respectivamente.

Solo Sonora logró obtener una calificación óptima, por encima de la media, en los cinco indicadores analizados. Le siguen un grupo de cinco entidades (Aguas-

22 La calificación otorgada a las entidades en el Indepol es la siguiente: mientras más cerca del o se encuentre la entidad mejor ha sido su cumplimiento de los lineamientos del SNSP. Para este artículo, a la calificación obtenida por cada entidad se le sumaron cien puntos, con el fin de estandarizarla a números positivos.

${ }^{23}$ El esfuerzo más cercano a una evaluación sistemática a las policías realizado por dicha autoridad fue un documento titulado "Modelo óptimo policial", en el que se evalúan varios rubros de la profesionalización policial a nivel estatal, pero no permite evaluar todos los rubros en forma conjunta ni hacer un comparativo entre entidades. 
calientes, Baja California Sur, Campeche, Oaxaca y Zacatecas) con cuatro de los cinco rubros con porcentajes por encima de la media. También destaca un tercer grupo de cinco entidades que, a pesar de tener menos de cuatro indicadores con resultados por encima de la media, presentan algunos de los mejores puntajes en uno o más de estos. Conforman este grupo Chihuahua, Jalisco, Nuevo León, Yucatán y Zacatecas. Esto significa que in de los gobiernos estatales lograron construir una policía que responde a las necesidades ciudadanas o que al menos estos tienen una percepción positiva de su trabajo. La Ciudad de México muestra un claro contraste con otras entidades y demuestra que el hecho de ejercer un mayor gasto y tener una enorme cantidad de policías no resulta en mejor capacidad. Por el contrario, es la peor evaluada por sus habitantes. El resto de los gobiernos, es decir, las dos terceras partes de la república mexicana siguen con carencias importantes en cuanto al servicio desplegado por sus policías.

Para la siguiente fase, las variables comprendidas en el cuadro 2 se sometieron a un análisis factorial, por medio del cual se pudo obtener un índice que aglutina todos los indicadores analizados anteriormente, y eso permite estimar las capacidades estatales tomando en cuenta cada una de las dimensiones discutidas. Los resultados obtenidos fueron los siguientes: ${ }^{24}$

${ }^{24}$ El tratamiento que se le dio a los datos fue el siguiente: en primer lugar, se realizó una matriz policórica con la cual se identificó que existieran correlaciones entre las variables. En segundo lugar, se practicó el análisis factorial con base en la matriz de correlaciones obtenida. La técnica de factores principales parte de un modelo hipotético en el que se asume que un número determinado de factores es mucho más pequeño que el número de variables que son responsables de las correlaciones observadas. En consecuencia, los nueve indicadores iniciales se agruparon en dos factores principales. En el primer factor quedaron las variables de percepción ciudadana: corrupción, desempeño, confianza y seguridad. En el segundo, las variables de gasto, estado de fuerza, el Indepol, así como las variables de motivo de la detención y debido proceso. Partiendo del supuesto de que los factores están asociados entre sí se les aplicó una rotación ortogonal. Una vez realizado ese proceso se creó un índice, tomando en cuenta las ponderaciones que los cálculos arrojaron para cada factor. 


\section{Gráfica 2}

Indice de capacidad estatal en seguridad pública 2016

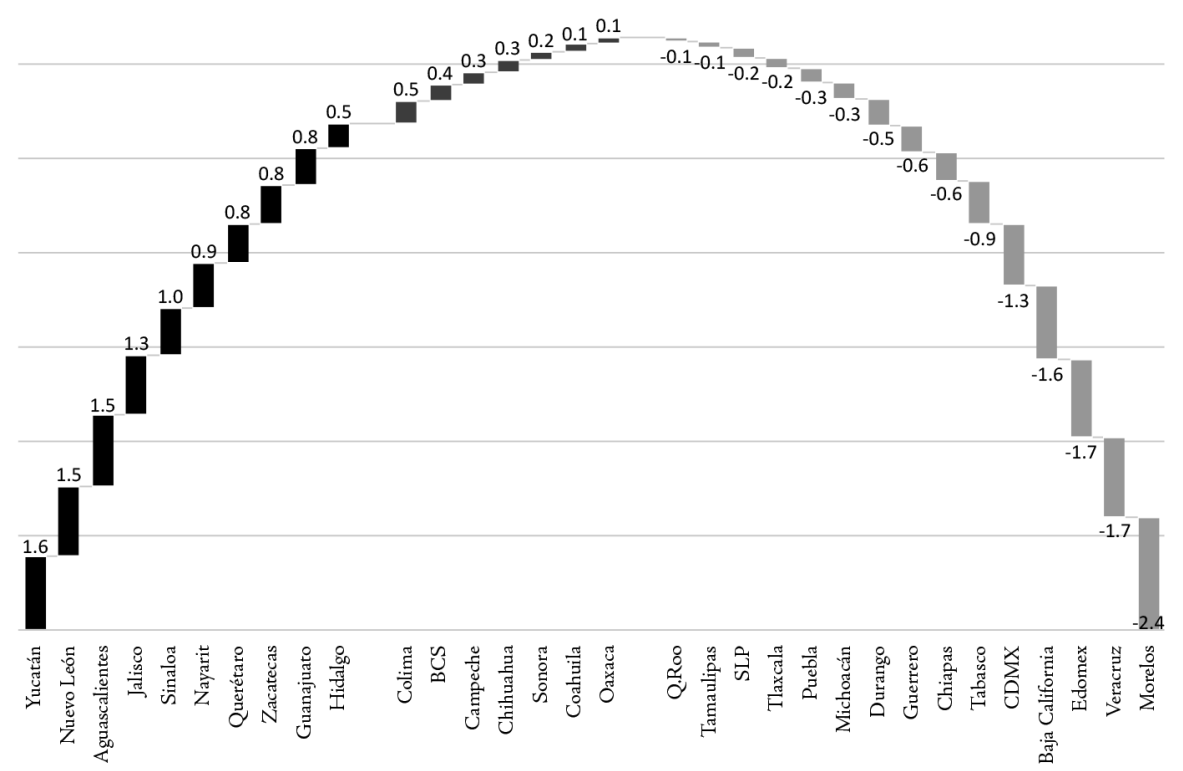

Fuente: Elaboración propia con base en los indicadores expuestos en el cuadro 2.

La gráfica 2 muestra tres grandes grupos: en primer lugar, del lado izquierdo y en color negro, se encuentra un grupo de diez estados, que son los que demostraron tener mayores capacidades para desplegar una política de seguridad pública en 2016. Esto pone de manifiesto que a pesar de que todas las policías presentan retos importantes para su consolidación, solo una tercera parte de ellas mostró mejoras. Destacan los casos de Yucatán y Nuevo León como los gobiernos estatales con capacidades en seguridad más desarrolladas. En el caso yucateco, una posible explicación del resultado favorable se encuentra en su estabilidad institucional, pues a diferencia de la mayoría de los estados, en 2016 la entidad experimentaba el noveno año consecutivo de su secretario de Seguridad Pública. En el caso de Nuevo León, el I4 de septiembre de zoII se graduó la primera generación de Fuerza Civil, proyecto basado en el programa de Policía Estatal Acreditable, que con adecuaciones e inversión de la iniciativa privada logró crear una institución sólida que se ha convertido en referente nacional. 
En segundo lugar, en medio de la imagen se encuentra un grupo de entidades que presentaron valores menores que el primer grupo, lo cual representa un desarrollo limitado de capacidades. Este grupo está integrado por siete entidades: Colima, Baja California Sur, Campeche, Chihuahua, Sonora, Coahuila y Oaxaca. Destacan los casos de Chihuahua y Coahuila, que poco antes de 2010 experimentaron reformas importantes. Chihuahua fusionó su Secretaría de Seguridad Pública con la Fiscalía, mientras que Coahuila refundó su policía e inició un nuevo programa después de los escándalos producidos por su Grupo de Armamento y Tácticas Especiales, mejor conocido como G.A.T.E.S. Sin duda los procesos de reforma y reacomodo institucional repercutieron en el servicio de las policías y son un ejemplo de cómo los cambios abruptos de rumbo en la política de seguridad afectan su desarrollo.

En tercer lugar se encuentra un grupo de 15 estados que obtuvieron valores negativos en el índice, lo cual de ninguna manera debe entenderse como una tendencia a la disminución de capacidades. De hecho, el valor obtenido únicamente refleja que desarrollaron mucho menos capacidades que el resto de las entidades o que no lo hicieron. Se trata de estados que presentan problemas de violencia crónica como Michoacán, Guerrero y Tamaulipas. Nuevamente la Ciudad de México resulta interesante para el análisis: como se aprecia en los resultados generales tiene un gasto en seguridad y un estado de fuerza muy por encima al resto del país, no obstante, la inversión no ha logrado impactar la percepción de los ciudadanos, quienes consideran a la policía como altamente ineficiente y proclive a la corrupción.

Destacan Veracruz, Estado de México y Morelos con los resultados más bajos. En el caso de Veracruz, a pesar del desarrollo de un proyecto de policía estatal, en cierta medida similar al de Nuevo León, no consiguió una buena percepción ciudadana. 2016 fue el último año de gobierno de Javier Duarte, una de las gubernaturas más polémicas en los últimos años, caracterizada por escándalos relacionados con las policías, acusadas de abusos sistemáticos y participación en desapariciones y ejecuciones extrajudiciales. En el caso del Estado de México, es bien conocida su trayectoria inconsistente en materia de seguridad, su incapacidad para consolidar una policía estatal y coordinarse con algunos de los municipios más importantes de la región metropolitana, como Ecatepec o Nezahualcóyotl.

Al último se encuentra Morelos, que en 2016 era gobernado por Graco Ramírez y como secretario de Seguridad Pública se encontraba Alberto Capella, quien en 2014 comenzó un proyecto de depuración de las policías, conformación de un mando único basado en el control de las fuerzas municipales por parte de la policía estatal, mientras que la entidad era azotada por una ola de violencia provocada por la dispersión de grupos del crimen organizado. Capella dimitió en 20I9, en un contexto 
de desgaste institucional provocado por las fricciones con los municipios, por el intento de conformación del mando, y porque fue acusado, ya en la gubernatura de Cuauhtémoc Blanco, de supuesto desvío de fondos durante la administración de Ramírez. La combinación de presencia del crimen organizado, policías con pocas habilidades, conflicto y falta de capacidad política para articular un proyecto de seguridad forman parte de la explicación que sitúa a la entidad como la peor calificada.

\section{Conclusiones}

Los hallazgos que presentamos representan un esfuerzo por hacer una comparación sólida de las policías estatales y clasificarlas según su capacidad institucional, y demuestran que dicha capacidad es resultado de la concurrencia de varios factores que dan cuenta de tres procesos de construcción institucional: el compromiso por forjar una policía profesional en las entidades, la relación del gobierno estatal con el federal y con los gobiernos municipales y el ejercicio eficiente del gasto en seguridad.

El índice muestra que las capacidades en seguridad pública de los estados son variadas y que los procesos de cambio no están determinados por el desarrollo de un solo factor. Los resultados apuntan a que, después de dos décadas de la transición democrática y de numerosas reformas a las organizaciones policiales, no se ha logrado construir una institución civil con la capacidad suficiente para enfrentar los problemas de seguridad pública en el marco de un estado de derecho. En las entidades federativas el panorama es sumamente diverso; encontramos que varias tuvieron avances en distintas dimensiones de capacidad, mientras que otras siguen rezagadas.

Como resultado del trabajo identificamos tres conjuntos de entidades con avances y retrocesos. En primer lugar, existe un grupo con serias dificultades o capacidades muy limitadas en seguridad pública. En segundo, figura un grupo con capacidades limitadas pero que puede operar, y finalmente se encuentra el grupo de estados que han mostrado mayor capacidad estatal en seguridad. El resultado es contrario a la intuición, pues el hecho de que una entidad tenga muchos recursos financieros o de fuerza policial no implica mayor fortaleza en nuestra medición, como lo reflejan la Ciudad de México y el Estado de México. Es importante hacer notar que esto de ninguna manera garantiza que los logros alcanzados puedan mantenerse sin continuidad, consistencia y optimización.

El trabajo contribuye con pruebas empíricas a las ideas de Polidano y Ostrom, así como parcialmente a los especialistas en fortalecimiento de las organizaciones 
policiales. Comprueba que la fuerza importa, igual que los acuerdos de coordinación y mando único mejoran el desempeño; que la calidad del trabajo policial medido en mejoras al entrenamiento, en profesionalización, en detenciones apegadas al debido proceso y evaluadas con satisfacción por la ciudadanía expresa mejoras y que estos indicadores superan los límites de las mediciones tradicionales del trabajo policial. Algunas políticas, como el control de confianza, surgida de las demandas ciudadanas por una policía confiable, sí alteraron la manera de actuar de las policías y las obligaron a rendir cuentas, pero los resultados son ambivalentes en ese procedimiento aislado.

El resultado también fortalece las ideas de otros enfoques de la literatura sobre organizaciones, tales como la del bote de basura. Concuerda con ella en las dificultades que enfrentan los regímenes políticos cuando intentan realizar reformas profundas, de largo plazo, con acuerdos limitados entre coaliciones de poder, sin un liderazgo consolidado y con metas no consensuadas. No obstante, también se comprueba que la fuerza policial no importa tanto per se, como dice la literatura policial, sino por su combinación con otros factores. Es igualmente vital que la ciudadanía perciba mejoras en el trabajo policial.

Entre las ventajas del índice propuesto están que resuelve los problemas de evaluación tradicionales de las políticas de seguridad, que llevan a concluir fracasos, más que logros. Lo que mostramos es un proceso inacabado, pero que claramente muestra que algunos gobiernos con sus respectivas policías han realizado transformaciones que están impactando de forma positiva en la percepción ciudadana.

Sobre el tema de gasto público podríamos concluir, de acuerdo con Polidano, que los estados han incrementado sus habilidades en el diseño e implementación de programas para crear políticas. Y aunque el uso del gasto se ha vuelto cada vez más detallado, las medidas de verificación y rendición de cuentas deben reforzarse para hacerlo más efectivo. También persiste un residuo de free riding, debido tal vez al modelo político.

La distribución de los factores confirma que el concepto de capacidad estatal en seguridad pública aglutina de manera correcta diferentes procesos, que las variables seleccionadas dan cuenta adecuadamente de ellos, y que están relacionadas entre sí. Además, sugiere algunas pistas para el análisis de relaciones entre variables en futuras investigaciones. Por ejemplo, será importante avanzar hacia un examen pormenorizado del gasto en seguridad de los estados, su relación con las fluctuaciones en los estados de fuerza y con la permanencia y el desarrollo de programas sólidos.

Otros indicadores de avance, aunque polémicos, son la instauración de los acuerdos del mando único, junto con el programa de Policía Estatal Acreditable. Habrá 
que preguntarse si el estado de fuerza es un producto de las tensiones entre federación, estados y sus policías.

Otra conclusión importante es la falta de coordinación y acuerdos. La federación ha tenido poca fuerza para comprometer y luego para hacer cumplir a los estados, y posiblemente estos a los municipios. El desarrollo de policías locales sucede en medio de una suerte de caos federal, estatal y municipal, producido por numerosas crisis de violencia y cambios reactivos sin la orientación consistente que debería buscarse en las reformas. Podemos además concluir que no todas las crisis policiales se originaron por el impacto del crimen organizado, sino que algunas son consecuencia de las reformas. Precisamente, los cambios federales anunciados por la nueva administración presidencial podrían abonar al desorden. La creación de la Guardia Nacional militarizada anunció cambios drásticos en las relaciones con los gobernadores, y la iniciativa de una nueva policía cívica aún está por determinar si será un modelo constructivo de avance para crear un estándar de policías de base o destruirá los desarrollos previos.

Las reformas policiales empiezan con muy buenos propósitos y terminan con muchas secuelas no previstas. En el trabajo mostramos algunas consecuencias de los continuos cambios y las transgresiones a las organizaciones policiales, al conjunto de reglas y procedimientos que afectan la construcción de una estatalidad en seguridad pública con numerosos obstáculos. Pero no todo ha sido fracasos. Varios gobiernos de los estados han preferido implantar soluciones propias al problema, tomando acciones para reconstruir sus policías.

Varios temas quedan pendientes de desarrollar para fortalecer este modelo de capacidades, entre ellos explicar cómo el ciclo político electoral de los gobiernos estatales puede estar afectando la construcción de capacidades; el efecto que tiene el cambio de administración presidencial y el anuncio de nuevas políticas centralizadas, así como el path dependence planteado para explicar las dificultades de los cambios.

Agradecemos a los dictaminadores anónimos del trabajo, así como a los organizadores del número de la revista sobre policías y seguridad por la oportunidad de publicar con ellos. 


\section{Referencias}

Aberbach, Joel y Tom Christensen

2013 "Why Reforms So Often Disappoint", American Review of Public Administration, 44 (I), pp. 3-16, https://doi.org/I0.1177\%2Fo275074013504I28

Aguayo, Quezada, Sergio y Jacobo Dayán

2018 El yugo Zeta: norte de Coabuila 2010-2011, México, El Colegio de México Alvarado, Mendoza, Arturo

2019 "Reformas policiales y la construcción de un Estado de derecho. El caso de la policía vecinal de Ciudad Nezahualcóyotl”, Desacatos, 60, pp. 34-57, https://doi.org/I0.29340/60.2089

Alvarado Mendoza, Arturo

2006 Estudio sobre seguridad pública en cuatro entidades mexicanas, Reporte para el Banco Mundial. s.p.i

Alvarado, Mendoza, Arturo

2012 El tamaño del infierno. Un estudio sobre la criminalidad en la Zona Metropolitana de la Ciudad de México, México, El Colegio de México.

Arroyo, Juárez Mario

2006 Estudio sobre seguridad pública en cuatro entidades mexicanas. El caso de Michoacán, Reporte para el Banco Mundial. s.p.i

Arzt, Sigrid

2006 Estudio sobre seguridad pública en cuatro entidades mexicanas. El caso de Chibuabua, Reporte para el Banco Mundial. s.p.i

Auditoría Superior de la Federación

2018 "Informe individual del Resultado de la Fiscalización Superior de la Cuenta Pública 20I8", https://www.asf.gob.mx/Section/58_Informes_de_auditoria

Bailey, John

2014 The Politics of Crime in Mexico: Democratic Governance in a Security Trap, Boulder, Colorado, FirstForumPress (a division of Lynne Rienner Publishers, Incorporated.

Benítez Manaut, Raúl y Sergio Aguayo Quezada (eds.) 2016 Atlas de la Seguridad y la Defensa de México, México, Instituto Belisario Domínguez-Senado de la República/ Colectivo de Análisis de la Seguridad y Democracia A.C. (Casede). 
Brandl, Steven, Mitchell Chamlin y James Frank

1995 "Aggregation bias and the capacity for formal crime control: The determinants of total and disaggregated police force size in Milwaukee, I934-1987", Justice Quarterly, I2(3), pp. 543-562, https://doi. org/I0.1080/07418829500096131

\section{Causa en Común}

20I8 Evaluación de la dinámica del gasto público en seguridad en México: el caso del FASp durante el periodo 2013-2018, http://causaencomun. org.mx/beta/wp-content/uploads/2019/o5/FASP-Investigacion-Completa-Final-230II9-I.pdf

Causa en Común

2017 Reporte de resultados del Índice de Desarrollo Policial (Indepol), https:// causaencomun.org.mx/beta/indepol-indice-de-desarrollo-policial/

Chamlin, Mitchell y Robert Langworthy

1996 "The police, crime, and economic theory: A replication and extension", American Journal of Criminal Justice, 20(2), pp. 165-182, https://doi. org/IO.1007/BFO2886924

Christensen Tom, Per Lægreid y Lise Rykkja

2017 "Reforming the Norwegian police between structure and culture: Community police or emergency police", Public Policy and Administration, 33(3), pp. I-I9.

Cohen, Michel, James March y Johan Olsen

1972 "A Garbage Can Model of Organizational Choice", Administrative Science Quarterly, I7(I), pp. I-25, http://doi.org//I0.2307/2392088

Correa-Cabrera, Guadalupe

2017 LOS Zetas InC.: Criminal Corporations, Energy, and Civil War in Mexico, Austin, University of Texas Press.

Dahl, Robert y Charles Lindblom

1953 Politics, Economics, and Welfare: Planning and Politico-Economic Systems Resolved into Social Processes, Nuev York, Harper and Brothers.

Davis, Diane E.

2006 "Undermining the rule of law: Democratization and the dark side of police reform in Mexico", Latin American Politics and Society, 48(I), pp. 55-86, https://doi.org/IO.IIII/j.1548-2456.2006.tboo338

Davis, Diane

2020 "Urban Violence and the Spatial Question: The Built Environmental. Correlates of (In) Security in Latin American Cities”, en Liliana Rivera 
y Xóchitl Bada (eds.), The Oxford Handbook of the Sociology of Latin America, Oxford, Oxford University Press, https://doi.org/ı..I093/ oxfordhb/9780190926557.013.4I

Diario Oficial de la Federación

2009 Ley General del Sistema Nacional de Seguridad Pública, Cámara de Diputados del H. Congreso de la Unión, http://www.diputados.gob.mx/ LeyesBiblio/pdf/LGSNSP_270519.pdf

Durán, Angélica

2020 "Illicit Drugs and Organized Crime in Latin America: New Scholarship and the Future of Alternative Policies", en Liliana Rivera y Xóchitl Bada (eds.), The Oxford Handbook of the Sociology of Latin America, http:// doi.org/10.1093/oxfordhb/9780190926557.013.43

García, Genaro

2006 ¿Por qué I 661 corporaciones de policía no bastan?, México, Kan Sasana Printer.

González, Yanilda

2019 "The Social origins of Institutional Weakness and Change: of Preferences, Power, and Police Reform in Latin America", World Politics, 7I(I), pp. 44-87, https://doi.org/I0.1017/Soo43887II8000I4X

Guerrero Gutiérrez, Eduardo

201 "Determinantes del subejercicio presupuestal en el Fondo de Aportaciones para la Seguridad Pública (FASP) del Ramo 33", en Carlos Chiapa y César Velázquez (coords.), Estudios del ramo 33, El Colegio de México, CONEVAL, pp. 227-270.

Instituto Nacional de Geografía y Estadística (INEGI) 2016 Encuesta Nacional de Población Privada de la Libertad (enPol), INEgI.

Instituto Nacional de Geografía y Estadística

2016 Encuesta Nacional de Victimización y Percepción sobre Seguridad Pública (ENVIPE), INEGI.

Instituto Nacional de Geografía y Estadística

2010-2018 Glosario de conceptos, Censo Nacional de Gobierno.

Kingdon, John y Eric Sotano

1984 Agendas, alternatives, and public policies, Little, Brown

Koper, Christopher, Gretchen E. Moore y Edward R. Maguire

$200 \mathrm{I} H i r i n g$ and Retention Issues in Police Agencies: Readings on the Determinants of Police Strength, Hiring and Retention of Officers, and the Federal cops Program, Urban Institute, Justice Policy Center. 
Langworthy, Robert $\mathrm{H}$.

I986 The structure of police organizations, Praeger Publisher.

Langworthy, Robert

1986 The structure of police organizations, Praeger Publisher.

LaRose, Antony P. y Sean A. Maddan

2009 "Reforming La Policía: looking to the future of policing in

Mexico", Police Practice and Research,Io(4), pp. 333-348, https://doi. org/10.1080/15614260802586327

Lindblom, Charles

1959 "The Science of "Muddling Through", Public Administration Review, I9(2), pp. 79-88, http://doi.org/I0.2307/973677

Lisón, Carlos

2016 "Reforma policial y gasto público en entidades federativas y municipios mexicanos (2008-2013)", Revista Policía y Seguridad Pública, 6(2), pp. 3I-80, https://doi.org/I0.5377/rpsp.v6i2.2989

Llanos, Luis Felipe, Luis Rivas, Fernando Lambarry y Mara Trujillo

2018 "La eficacia de la policía estatal en México", Perfiles latinoamericanos, 26(52), pp. I-26, http://dx.doi.org/I0.18504/pl2652-015-2018

Luna Leal, Marisol

2014 "La implementación del modelo de policía estatal acreditable con mando único coordinado y su incidencia en los municipios mexicanos; actualización y avances", Revista legislativa de estudios sociales y de opinión pública, 7(13), pp.73-98.

Maguire, Edward y Craig Uchida

2000 "Measurement and Explanation in the Comparative Study of American Police Organizations", Criminal justice, 4, pp. 49I-557.

Maldonado, Salvador

2017 "Transformaciones de la violencia y la criminalidad mexicana. Solidaridad forzada y violencia silenciosa desde la periferia”, en Santiago Álvarez, Brígida Renoldi y Salvador Maldonado (comps.), Estado, violencia y mercado. Conexiones etnográficas en América Latina, Antropofagia editores, pp. 23I-252.

México Evalúa, Centro de Análisis de Políticas Públicas

2018 Arquitectura del Ramo 23, México Evalúa, http://www.mexicoevalua. org/numerosdeerario/wpcontent/uploads/2017/03/2018_02_27_ Caja_Negra2.o_Documento.pdf 
México Evalúa, Centro de análisis de políticas públicas

20II El gasto en seguridad: Observaciones de la Auditoría Superior de la Federación a la gestión y uso de recursos, Secretaría de Hacienda y Crédito Público, Cuenta Pública, ejercicios fiscales 2013-2017.

Moloeznik, Marcos y María Eugenia Suárez

2012 "El proceso de militarización de la seguridad pública en México (20062010)", Frontera Norte, 24(48), pp. I2I-I44.

Morales, Sabina y Carlos Pérez

2015 "La militarización, un obstáculo para la gobernanza democrática de la seguridad en México", Revista colombiana de sociología, 38(I), pp. 83-I03, http://dx.doi.org/IO.15446/rcs.v38ni.53279

Muedano, Marcos

2018 "Faltan a México 96 mil policías; incumple estándar de la onU", Excélsior, https://www.excelsior.com.mx/nacional/faltan-a-mexico-96-milpolicias-incumple-estandar-de-la-onu/126203I

Olson, Mancur

1965 The Logic of Collective Action. Public Goods and the Theory of Groups, euA, Harvard University Press.

Organización de las Naciones Unidas (ONU)

2017 Police Capacity-Building and Development, Department of Peacekeeping Operations, https://police.un.org/sites/default/files/sgf-guidelines_ police_cbd-2015.pdf

Ostrom, Elinor

2005 "Understanding the Diversity of Structured Human Interactions", en Elinor Ostrom (ed.), Understanding Institutional Diversity, Princeton, Princeton University Press.

Ostrom, Elinor

2000 El Gobierno de los Bienes Comunes: La Evolución de las Instituciones de Acción colectiva, México, Universidad Nacional Autónoma de México.

Padilla, Sergio

2019 "Los límites de la militarización de la seguridad pública en México", Animal Politico, https://www.animalpolitico.com/el-blog-de-causa-en-comun/los-limites-a-la-militarizacion-de-la-seguridad-publica-en-mexico/

Polidano, Charles

2000 "Measuring public sector capacity", World Development, 28(5), pp. 805822, https://doi.org/ı0.1016/So305-750X(99)00158-8

Sabet, Daniel 
2012 Police Reform in Mexico: Informal Politics and the Challenge of Institutional Change, Stanford University Press.

Sánchez, José Said

2020 "Gasto subnacional, presupuestado y ejercido: la brecha entre el plan y la práctica en México (2003-2016)", Foro Internacional, I, pp. 139-174, https://doi.org/I0.2420I/fi.v60iI.26II

Schedler, Andreas

2018 En la niebla de la guerra: Los ciudadanos ante la violencia criminal organizada, México, CIDE.

Silva, Forné, Carlos, Catalina Pérez Correa y Rodrigo Gutiérrez Rivas

2018 "Use of Lethal Force by Mexican Security Forces 2007- 2015", en Alejandro Anaya-Muñoz y Barbara Frey (eds.), Mexico's Human Rights Crisis, University of Pennylvania Press, pp. 23-42.

Silva Forné, Carlos y Sergio Padilla Oñate

2020 "Tortura en la guerra contra el narcotráfico en México, entre la militarización, el control político y el nuevo sistema de justicia penal", Latin American Law Review, 4, pp. I07-I28, https://doi.org/I0.29263/ laro4.2020.05

Simon, Herbert

1957 Models of Man: Social and Rational; Mathematical Essays on Rational Human Behavior in Society Setting, Wiley.

Sirnes Hjelum, Magnus y Per Lægreid

2019 "The challenge of transboundary coordination: The case of the Norwegian police and military", Safety Science, II5, pp. I3I-I40, https://doi. org/IO.IOI6/j.ssci.2019.0I.027

Solís, Vanesa

2019 "Ellos son los gobernadores que más faltan a reuniones de seguridad", El Sol de México, https://www.elsoldemexico.com.mx/mexico/sociedad/ellos-son-los-gobernadores-que-mas-faltan-a-reuniones-de-seguridad-4596668.html

Stucky, Thomas

2005 "Local politics and police strength", Justice quarterly, 22(2), pp. 139-169, https://doi.org/I0.1080/07418820500088739

Suárez, María Eugenia, Marcos Moloeznik y David Shirk

201 Justiciabarómetro. Diagnóstico Integral de la Policía Municipal en Ciudad Juárez, Justice in Mexico Project/ El Colegio de Chihuahua/ El Colegio de la Frontera Norte/Universidad de San Diego. 
Ungar, Mark

2002 Elusive Reform: Democracy and the rule of law in Latin America, Lynne Rienner Publishers.

Urbina, Gustavo y Sergio Bárcena

2019 Herramientas de análisis multivariado para la investigación social. Una guía práctica en stata, México, Tecnológico de Monterrey.

Wilson, James

1968 "The Urban Unease: Community vs. City", The Public Interest, I2, pp. 25-39.

Wilson, James y Barbara Boland

1978 "The effects of the police on crime", Law E Society Review, I2(3), pp. 367-390, https://doi.org/I0.2307/3053285

Arturo Alvarado

Profesor-investigador del Centro de Estudios Sociológicos de El Colegio de México, doctor en Sociología por el Colegio de México, especialista en seguridad, derechos humanos y gobernanza democrática.

Sergio Padilla Oñate

Profesor de la especialidad en seguridad pública de la Facultad de Ciencias Políticas y Sociales de la Universidad Nacional Autónoma de México. Candidato a doctor en Ciencias Sociales con especialidad en Sociología por El Colegio de México, maestro en Sociología Política por el Instituto de Investigaciones Dr. José María Luis Mora. Licenciado en Sociología por Facultad de Ciencias Políticas y Sociales de la Universidad Nacional Autónoma de México.

Citar como: Arturo Alvarado, Sergio Padilla Oñate (202I), "Organización policial y debilidad institucional: balance de las capacidades de las policías estatales", Iztapalapa. Revista de Ciencias Sociales y Humanidades, núm. 90, año 42, enero-junio de 202I, ISSN: 2007-9176; pp. II-47. Disponible en $<$ http://revistaiztapalapa.izt.uam.mx/index.php/izt/issue/archive $>$. 